\title{
Intoxicação espontânea de bovinos por Senna obtusifolia no Estado do Paraná ${ }^{1}$
}

\author{
Gustavo R. Queiroz ${ }^{2}$, Rita C.L. Ribeiro ${ }^{3}$, Fernanda T.N.M.A. Romão ${ }^{4}$, \\ Karina K.M.C. Flaiban ${ }^{5}$, Ana Paula F.R.L. Bracarense ${ }^{5}$ e Júlio A.N. Lisbôa ${ }^{6 *}$
}

\begin{abstract}
Queiroz G.R., Ribeiro R.C.L., Romão F.T.N.M.A., Flaiban K.K.M.C., Bracarense A.P.F.R.L. \& Lisbôa J.A.N. 2012. [Spontaneous Senna obtusifolia poisoning in cattle in the state of Parana, Brazil.] Intoxicação espontânea de bovinos por Senna obtusifolia no estado do Paraná. Pesquisa Veterinária Brasileira 32(12):1263-1271. Departamento de Clínicas Veterinárias, Centro de Ciências Agrárias, Universidade Estadual de Londrina, Campus Universitário, Cx. Postal 6001, Londrina, PR 86051-990, Brazil. E-mail: janlisboa@uel.br

Plants of the genus Senna cause a degenerative myopathy in cattle and most of the reports refer to Senna occidentalis. The aim of this paper is to report, for the second time in Brazil, an outbreak of natural poisoning by Senna obtusifolia. It happened in the northwestern Paraná in a herd of 200 cows, 45 to 152 months of age, which had been placed into a feedlot to improve their nutritional status before the calving period. The cows stayed for eight days in this feedlot infested by the plant. Four to nine days after they got into the feedlot, 20 cows became ill and only one recovered. The clinical signs consisted of myoglobinuria, incoordination and permanent sternal recumbency. The affected cows showed increased activity of creatine phosphokinase, aspartate aminotransferase, gama-glutamyltransferase, and alkaline phosphatase. The main postmortem changes were in skeletal muscles of the hind limbs characterized by pale areas. The histological alterations were multifocal segmental necrosis of skeletal muscles and hepatic multifocal paracentral necrosis. Epidemiological, clinical and pathological data led to the diagnosis of Senna obtusifolia poisoning. The plant showed miotoxic and hepatotoxic effects on the poisoned animals and the disease was almost always lethal.
\end{abstract}

INDEX TERMS: Poisonous plants, Senna obtusifolia, muscle necrosis, serum biochemistry, myopathy, plant poisoning, diseases of cattle.

RESUMO.- Plantas do gênero Senna causam miopatia degenerativa em bovinos e o maior número de relatos envolve Senna occidentalis. 0 objetivo desse trabalho é relatar, pela segunda vez no Brasil, um surto de intoxicação natural por

\footnotetext{
${ }^{1}$ Recebido em 11 de agosto de 2012.

Aceito para publicação em 17 de setembro de 2012.

Dissertação de Mestrado do primeiro autor pelo Programa de Pós-Graduação em Ciência Animal da Universidade Estadual de Londrina (UEL).

${ }^{2}$ Mestre em Ciência Animal, UEL. Endereço particular: Rua Raposo Tavares 1074, Apto 104, Londrina, PR 86010-580, Brasil.

${ }^{3}$ Hospital Veterinário, Universidade Paranaense (Unipar), Rodovia PR $480 \mathrm{Km}$ 2, Umuarama, PR 87502-970, Brasil.

${ }^{4}$ Mestrando em Ciência Animal, UEL. Endereço particular: Rua Maranhão 209, Apto 73, Londrina, PR 86010-410.

${ }^{5}$ Departamento de Medicina Veterinária Preventiva, Centro de Ciências Agrárias (CCA), UEL, Londrina, PR.

${ }^{6}$ Departamento de Clínicas Veterinárias, CCA-UEL, Campus Universitário, Cx. Postal 6001, Londrina, PR 86051-990l. *Autor para correspondência: janlisboa@uel.br
}

Senna obtusifolia. É descrito um surto de intoxicação por Senna obtusifolia, na região noroeste do estado do Paraná, que aconteceu em um lote de 200 vacas, com idade entre 45 e 152 meses, introduzidas em um confinamento para melhorar a condição corporal antes do parto. A área do confinamento estava invadida pela planta e as vacas permaneceram no local durante oito dias. Entre quatro e nove dias após a entrada no confinamento 20 vacas adoeceram e somente uma se recuperou. Os sinais consistiram em mioglobinúria, incoordenação e decúbito esternal permanente. As vacas doentes apresentaram aumento das atividades das enzimas creatina quinase, aspartato aminotransferase, gamaglutamiltransferase e fosfatase alcalina. Lesões na musculatura esquelética dos membros posteriores caracterizadas por áreas pálidas representaram as principais alterações observadas à necropsia de quatro bovinos. $\mathrm{Ne}$ crose segmentar multifocal da musculatura esquelética e necrose paracentral multifocal no fígado foram as altera- 
ções histopatológicas mais relevantes. As evidências epidemiológicas, clínicas e patológicas indicam o diagnóstico de intoxicação por Senna obtusifolia. A planta demonstrou ter efeito miotóxico e hepatotóxico nos animais intoxicados e a doença foi quase sempre fatal.

TERMOS DE INDEXAÇÃO: Plantas tóxicas, Senna obtusifolia, necrose muscular, bioquímica sérica, miopatia, intoxicação por plantas, doenças de bovinos.

\section{INTRODUÇÃO}

Plantas do gênero Senna, são importantes causadoras de intoxicação em bovinos caracterizada por miopatia degenerativa, mioglobinúria e morte (Henson et al. 1965). A intoxicação por Senna occidentalis já foi descrita nos Estados Unidos (Pierce \& O'Hara 1967, Schmitz \& Denton 1977), na Austrália (Rogers et al. 1979), e em Cuba (Marrero et al. 1998). No Brasil, a intoxicação natural de bovinos por $S$. occidentalis foi observada principalmente no estado do Rio Grande do Sul (Barros et al. 1990, 1999, Raffi et al. 2003, Carmo et al. 2011, Takeuti et al. 2011).

As intoxicações normalmente ocorrem no final do outono e no início do inverno, quando começa a existir escassez de pasto (Barros et al. 1999, Carmo et al. 2011, Takeuti et al. 2011). A intoxicação pode ocorrer por ingestão natural da planta ou ser acidental pela contaminação do concentrado com suas sementes (Barros et al. 1990). Podem se intoxicar bovinos de várias idades (Raffi et al. 2003). As intoxicações podem ocorrer em forma de surtos afetando até $50 \%$ do rebanho ou como casos isolados (Carmo et al. 2011). Os principais sinais clínicos são dificuldade de locomoção, decúbito esternal permanente, mioglobinúria e morte, e as lesões encontradas são principalmente localizadas na musculatura esquelética caracterizando a miopatia tóxica degenerativa aguda (Barros et al. 1990,1999, Raffi et al. 2003, Carmo et al. 2011, Takeuti et al. 2011).

Em comparação ao grande número de informações existentes sobre intoxicações por $S$. occidentalis, há poucos relatos sobre a intoxicação de ocorrência natural por $S$. obtusifolia em bovinos (Nicholson et al. 1977, McCormack \& Neisler 1980). No Brasil há apenas um relato de surtos ocorridos em Santa Catarina, o qual foi complementado pela intoxicação induzida experimentalmente (Froehlich 2010).

S. obtusifolia é conhecida popularmente como fedegoso, fedegoso-branco e mata-pasto liso. É uma planta perene, subarbustiva, lenhosa, ereta, medindo entre 70 e $160 \mathrm{~cm}$ de altura. Possui folhas alternadas e reprodução por sementes. Sua inflorescência é terminal e axilar, em racemos com poucas flores de coloração amarela e vagens quase cilíndricas, recurvadas de 10 a $20 \mathrm{~cm}$ de comprimento. Comporta-se como planta invasora de pastagens e lavouras e também é muito encontrada na beira de estradas (Lorenzi 1991).

Os princípios tóxicos de $S$. obtusifolia são várias antraquinonas (Lewis \& Shibamoto 1989) e um princípio catártico (Putnam et al. 1988). 0 componente miotóxico parece ser uma mistura de nove antraquinonas e três antronas (Lewis \& Shibamoto 1989).

Em virtude da escassez de informações disponíveis na literatura e da existência de apenas um único registro no país, o objetivo deste trabalho foi descrever aspectos epidemiológicos, clínicos e patológicos observados em um surto de intoxicação em bovinos no estado do Paraná, região Sul do Brasil.

\section{MATERIAL E MÉTODOS}

O surto de intoxicação ocorreu em uma propriedade rural localizada no município de Paranavaí, região noroeste do estado do Paraná. Teve início no final de julho de 2010, quando um lote de 200 vacas da raça Nelore em terço final de gestação, com idade entre 45 e 152 meses $(64,4 \pm 32,5$ meses), foi colocado em um confinamento como medida de manejo para melhorar sua condição corporal para o período de parição. A área de confinamento estava intensamente infestada por Senna obtusifolia (Fig.1). Uma vaca doente foi encaminhada para o Hospital Veterinário da Universidade Estadual de Londrina (HV-UEL) e a evolução da sua enfermidade foi acompanhada durante 6 dias. Os dados epidemiológicos e clínicos foram obtidos durante visita à propriedade, realizada oito dias após o início do surto. Exemplares da planta suspeita foram colhidos para classificação botânica e 15 vacas acometidas ainda vivas foram examinadas e delas foram colhidos os materiais para os exames laboratoriais.

Amostras de sangue foram colhidas por meio de punção da veia jugular com uso de frascos a vácuo sem anticoagulante, para a obtenção posterior do soro, e de frascos contendo EDTA para os exames hematológicos. As colheitas de líquor foram realizadas por punção no espaço atlanto-occipital, utilizando-se a agulha metálica do cateter intravenoso ${ }^{7} 18 \mathrm{G}$ com $5 \mathrm{~cm}$ de comprimento. A colheita foi realizada por gotejamento espontâneo desprezando-se o volume inicial de $3 \mathrm{~mL}$. Todo o material foi mantido refrigerado até a chegada ao laboratório.

Para o exame necroscópico, foram selecionadas três vacas acometidas, com 4 a 6 dias de evolução, que se apresentavam deprimidas e em decúbito lateral permanente. Adotou-se o seguinte protocolo para a eutanásia: sedação com xilazina $2 \%^{8}$, na dose de $0,2 \mathrm{mg} / \mathrm{kg}$; anestesia geral com tiopental ${ }^{9}$, na dose de $10 \mathrm{mg} / \mathrm{kg}$; e indução da parada cardíaca com solução de $\mathrm{KCl} 20 \%$ em volume variável de 250 a $350 \mathrm{~mL}$ infundido de forma rápida e contínua. Todos os fármacos foram administrados pela via intravenosa. A vaca internada no HV-UEL foi submetida à eutanásia e necropsiada com 9 dias de evolução estacionária e sem perspectivas de melhora clínica.

Foram colhidos, para exame histopatológico, fragmentos dos seguintes músculos: semimembranoso, semitendinoso, glúteo, vasto lateral, vasto medial, vasto intermédio, reto femural, ileopsoas, longíssimo dorsal, supraespinhoso, tríceps braquial, deltoide e infraespinhoso; além de miocárdio, baço, linfonodos, fígado, abomaso, pulmão, rim, intestino e sistema nervoso central, incluindo gânglio do nervo trigêmeo, rete mirabile carotídea e hipófise. Os fragmentos foram fixados em solução de formalina tamponada a $10 \%$.

Os componentes do eritrograma e a contagem total de leucócitos foram determinados em aparelho hematológico ${ }^{10}$, e a contagem diferencial de leucócitos foi realizada em esfregaços

\footnotetext{
${ }^{7}$ BD Angiocath TM, Becton Dickinson Ind. Cir. Ltda, Av. Presidente Juscelino Kubitschek, 273, Juiz de Fora, MG.

${ }^{8}$ Copazine ${ }^{\circledR}$, Schering-Plough Saúde Animal Ind. Com. Ltda, Av. Sir Harry Wellcome 335, Cotia, SP.

${ }_{9}$ Thiopentax $\AA$, Cristália Produtos Químicos Farmacêuticos Ltda, Rod. Itapira-Lindóia, Km 14, Itapira, SP.

${ }^{10}$ MS4® Melet Schloesing Laboratoires, Laborsys, Av. Das Torres 824, São José dos Pinhais, PR.
} 

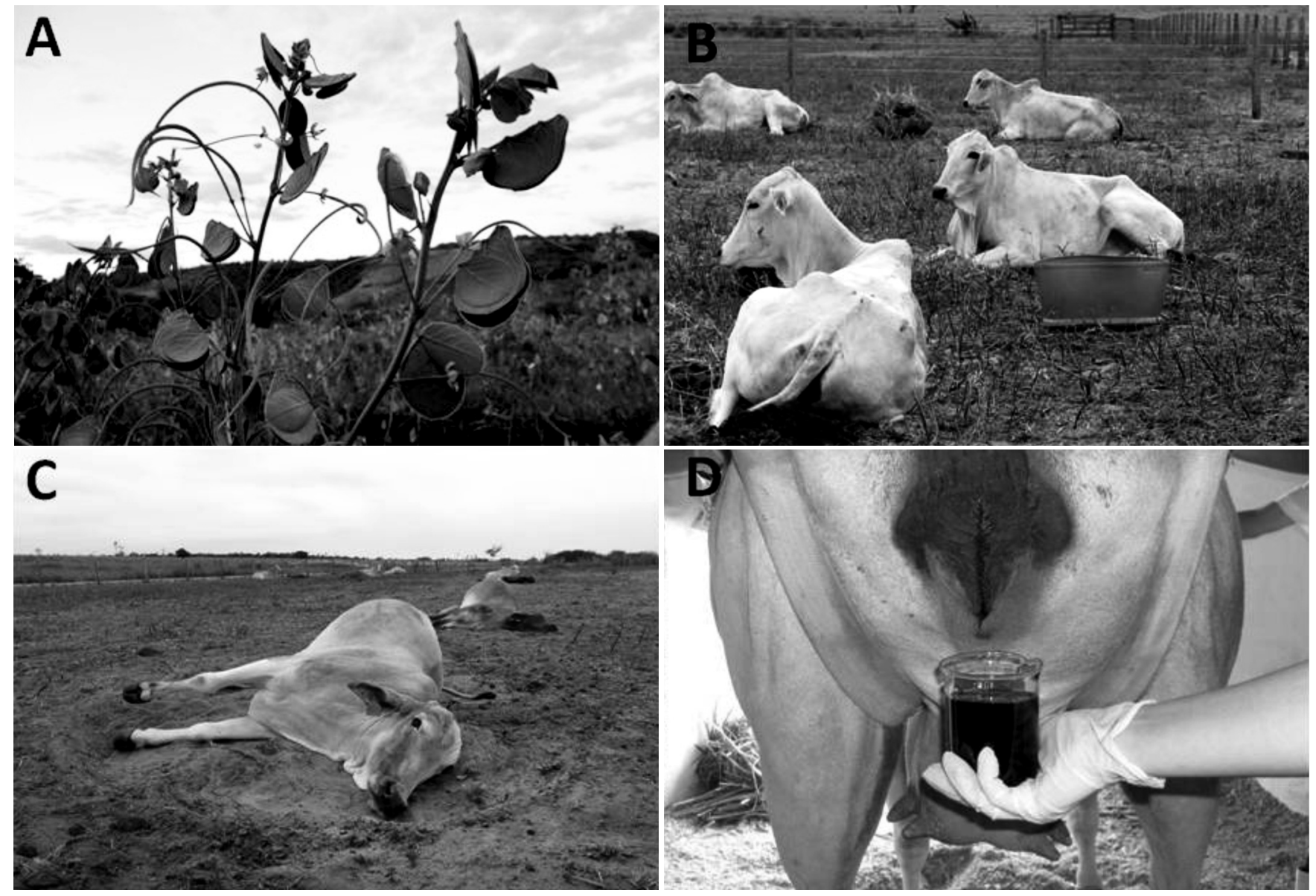

Fig.1. Senna obtusifolia. (A) Planta subarbustiva, perene, que possui flores de coloração amarelada e vagem cilíndrica e recurvada. (B) Vacas intoxicadas por S. obtusifolia em decúbito esternal permanente e alertas ou (C) em decúbito lateral permanente e deprimidas. (D) A hemoglobinúria foi relatada como manifestação comum nas vacas acometidas..

sanguíneos corados pela técnica de Romanowsky ${ }^{11}$, conforme recomendado por Jain (1993). A determinação da concentração de proteína plasmática total (PPT) foi realizada por refratometria e a concentração do fibrinogênio plasmático foi determinada pelo método da precipitação pelo calor seguido de leitura refratométrica (Kaneko \& Smith 1967).

As atividades séricas das enzimas gamaglutamiltransferase (GGT), aspartato aminotrasferase (AST), creatina quinase (CK) e fostatase alcalina (FA), e as concentrações de ureia e de creatinina foram mensuradas por técnicas cinéticas a $37^{\circ} \mathrm{C}$. As mensurações das concentrações de proteína total (PT), da albumina e das bilirrubinas total (BT) e direta (BD) no soro sanguíneo foram realizadas por métodos colorimétricos. Empregaram-se reagentes comerciais específicos ${ }^{12}$ e leitura espectrofotométrica utilizado aparelho de bioquímica úmida ${ }^{13} \mathrm{~A}$ concentração da bilirrubina indireta (BI) foi calculada por subtração entre os valores de BT e BD.

No líquor foram avaliadas as características físico-químicas e as contagens global e diferencial de células nucleadas e de hemácias. A contagem global de células foi realizada em câmara de Fuchs-Rosenthal (Stöber 1993) e a contagem diferencial em esfregaço corado pela técnica de Romanowsky ${ }^{11}$. As concentrações de proteína e de glicose foram determinadas por método colori-

\footnotetext{
${ }^{11}$ Panótico Rápido ${ }^{\circledR}$ Laborclin Produtos para Laboratórios Ltda, Rua Cassemiro de Abreu 521, Pinhais, PR.

${ }^{12}$ Dialab Diagnóstico S/A, Praça Carlos 49, $3^{\circ}$ andar, Belo Horizonte, MG.

${ }^{13}$ BS120® Mindray Chemistry Analyzer, JR Ehlke \& Cia Ltda, Av. João Gualberto 1661, Juvevê, Curitiba, PR.
}

métrico com leitura espectrofotométrica ${ }^{13}$, empregando-se reagentes comerciais específicos ${ }^{12}$.

Os fragmentos de tecido fixados em formol foram submetidos à desidratação em soluções crescentes de álcoois, diafanização em xilol e inclusão em parafina. Cortes de $5 \mu \mathrm{m}$ de espessura foram corados pelo método de hematoxilina-eosina (HE).

Para apresentação das variáveis hematológicas e liquóricas aplicou-se estatística descritiva com determinação das medidas de tendência central e de dispersão. Para avaliação das variáveis bioquímicas séricas as vacas foram agrupadas, conforme o padrão de comportamento que exibiam, em alertas $(n=10)$ e deprimidas $(n=6)$. 0 teste $t$ foi empregado para comparação entre os grupos, admitindo-se probabilidade de erro de 5\% (Curi 1998).

\section{RESULTADOS}

Das 200 vacas componentes do lote que ingressou no confinamento, 20 vacas adoeceram e somente uma se recuperou. As vacas permaneceram confinadas, em contato com a planta tóxica, durante 8 dias seguidos, e os casos ocorreram entre quatro e nove dias após a entrada no confinamento. Oito dias após a entrada, havia 14 vacas doentes e todo o lote foi retirado da área, retornando ao pasto. As seis últimas vacas apresentaram os sinais no pasto e a que se recuperou estava entre essas. Os sinais relatados eram incoordenação motora, urina vermelho enegrecida e evolução para o decúbito esternal permanente em até 12 horas, 
mantendo-se alertas e com apetite nos primeiros dias da doença.

A área do confinamento consistia em um piquete de $5.000 \mathrm{~m}^{2}$, com solo arenoso e intensamente infestada por Senna obtusifolia (Fig.1), cuja identidade botânica foi confirmada posteriormente, como base em critérios morfológicos. A planta estava presente em grande quantidade como a única espécie invasora e se distribuía, de maneira não uniforme, por toda a área do piquete. A ausência de folhas e de vagens e a presença de talos cortados eram evidências claras de que a planta tinha sido ingerida pelas vacas. A presença de S. obtusifolia se restringia à área do confinamento e exemplares não foram observados invadindo a pastagem.

Após confinadas, as vacas não sofreram privação de alimentos pois a oferta de volumoso (cana-de-açúcar triturada) acrescentado de concentrado (farelo de trigo e farelo de soja) foi garantida em cocho com extensão suficiente para evitar competição pelo espaço $(40 \mathrm{~cm}$ lineares/cabeça). Os motivos para a ingestão da planta invasora não foram esclarecidos completamente. Seria possível suspeitar que algumas vacas sem experiência prévia em sistema de confinamento tenham deixado de se aproximar do cocho e devido à fome, ingerido maior quantidade da planta disponível, o suficiente para intoxicarem-se.

Durante a visita à propriedade foram encontradas 15 vacas doentes e duas vacas mortas no confinamento. No pasto havia uma vaca doente, que não foi examinada, e outra se recuperando. As vacas examinadas apresentavam três a cinco dias de evolução. Em uma das vacas que morreram naturalmente a evolução durou 8 dias. A doença foi caracterizada por fraqueza muscular seguida por decúbito permanente. Nove vacas apresentavam-se em decúbito esternal, alertas e mantinham ingestão de água e alimentos (Fig.1). Cinco vacas estavam em decúbito lateral e deprimidas (Fig.1). A única vaca deprimida que ainda mantinha o decúbito esternal adotava a posição de auto-auscultação com a cabeça voltada para o flanco. A alteração do comportamento não estava relacionada com o tempo de evolução. Cinco das seis vacas deprimidas estavam doentes há quatro dias e a outra há cinco dias. A vaca que ficou internada teve a sua evolução acompanhada até o nono dia e permaneceu em decúbito esternal com quadro estacionário mantendo-se alerta e ingerindo alimentos e água. Era incapaz de sustentar o próprio peso quando erguida artificialmente.

$\mathrm{Na}$ avaliação das funções vitais, quatro vacas apresentaram bradicardia $(46,0 \pm 6,9 \mathrm{bpm})$, cinco apresentaram taquicardia $(102,4 \pm 16,4 \mathrm{bpm})$ e os outros sete animais tinham a frequência cardíaca normal $(66,8 \pm 6,4 \mathrm{bpm})$. Alteração do rítmo cardíaco foi observada em seis vacas. A frequência respiratória estava dentro do intervalo fisiológico $(27,0 \pm 7,1 \mathrm{mpm})$. Oito vacas apresentaram motilidade ruminal diminuída $(2,1 \pm 1,0 \mathrm{MR} / 5 \mathrm{~min})$ e as outras exibiam atonia ruminal. Sete vacas estavam hipotérmicas $\left(37,2 \pm 0,7^{\circ} \mathrm{C}\right)$ e as outras nove apresentavam-se normotérmicas $\left(38,5 \pm 0,3^{\circ} \mathrm{C}\right)$. Cinco das sete vacas hipotérmicas eram também deprimidas.

0 tônus da cauda e o tônus da língua eram diminuídos em dez e três vacas, respectivamente. Apenas dois animais apresentavam tremores musculares na região do pescoço e nos membros posteriores. As mucosas estavam congestas em sete animais. Quatorze bovinos tinham as fezes ressecadas e os outros dois apresentavam diarréia com fezes enegrecidas. Seis vacas apresentavam mioglobinúria eliminando urina com coloração vermelho enegrecida e quatro dessas eram também deprimidas. A vaca que permaneceu internada sob observação eliminou urina escura durante toda a evolução de sua doença (Fig.1).

Não foram observadas alterações no eritrograma (Quadro 1). Metade das vacas exibia leucopenia, três apresenta-

Quadro 1. Variáveis do hemograma de 16 vacas espontaneamente intoxicadas por Senna obtusifolia

\begin{tabular}{|c|c|c|c|c|c|c|}
\hline Variáveis & $\bar{\chi}$ & $\mathrm{s}$ & $\mathrm{Md}$ & $\mathrm{P}_{25}$ & $\mathrm{P}_{75}$ & $\begin{array}{l}\text { Valores de } \\
\text { referência }\end{array}$ \\
\hline $\mathrm{He}\left(\mathrm{x} 10^{6} / \mu \mathrm{l}\right)$ & 7,05 & 0,77 & 7,14 & 6,48 & 7,47 & $6,0-11,6^{*}$ \\
\hline $\mathrm{Hb}(\mathrm{g} / \mathrm{dL})$ & 14,26 & 1,48 & 14,20 & 13,33 & 15,40 & $8,5-16,5^{*}$ \\
\hline VG (\%) & 38,31 & 3,66 & 39,50 & 35,30 & 40,38 & 27-48* \\
\hline VCM (FL) & 54,58 & 2,20 & 54,75 & 53,55 & 56,20 & $30,5-55,5^{*}$ \\
\hline HCM (PG) & 20,26 & 1,52 & 20,75 & 19,15 & 21,35 & $9,7-19,0 *$ \\
\hline CHCM (\%) & 37,03 & 2,16 & 37,85 & 34,65 & 39,00 & $28,0-38,0^{*}$ \\
\hline $\begin{array}{l}\text { Leucócitos } \\
\left(\times 10^{3} / \mu \mathrm{L}\right)\end{array}$ & 8,06 & 4,58 & 6,05 & 4,88 & 10,07 & $6,2-12,2^{* *}$ \\
\hline $\begin{array}{l}\text { Segmentados } \\
\left(\mathrm{x} 10^{3} / \mu \mathrm{L}\right)\end{array}$ & 4,58 & 4,22 & 3,18 & 1,58 & 7,60 & $1,3-3,4^{* *}$ \\
\hline $\begin{array}{l}\text { Linfócitos } \\
\left(\times 10^{3} / \mu \mathrm{L}\right)\end{array}$ & 3,35 & 3,20 & 2,37 & 1,70 & 3,58 & $3,3-8,0 * *$ \\
\hline $\begin{array}{l}\text { Monócitos } \\
\left(\mathrm{x} 10^{3} / \mu \mathrm{L}\right)\end{array}$ & 0,09 & 0,09 & 0,06 & 0,01 & 0,12 & $0,0-0,5^{* *}$ \\
\hline $\begin{array}{l}\text { Fibrinogênio } \\
\text { (mg/dL) }\end{array}$ & 1268,75 & 359,11 & 1200,00 & 1000,00 & 1600,00 & $300-700^{* * *}$ \\
\hline PPT (g/dL) & 8,28 & 0,40 & 8,30 & 8,00 & 8,45 & $6,6-7,8^{* * *}$ \\
\hline
\end{tabular}

${ }^{*}$ Ayres et al. (2001), ${ }^{* *}$ Fagliari et al. (1998), ${ }^{* * *}$ Radostits et al. (2007).

Quadro 2. Variáveis bioquímicas no soro sanguíneo das vacas espontaneamente intoxicadas por Senna obtusifolia que se apresentavam alertas $(n=10)$ ou deprimidas $(n=6)$

\begin{tabular}{|c|c|c|c|}
\hline Variáveis & Depressão & $\bar{\chi} \pm \mathrm{s}$ & $\begin{array}{c}\text { Intervalo de varia- } \\
\text { ção fisiológica }\end{array}$ \\
\hline \multirow[t]{2}{*}{ CK (UI/L) } & não & $18.910,00^{\mathrm{a}} \pm 3.850,02$ & $35-280 *$ \\
\hline & $\operatorname{sim}$ & $16.490,00^{\mathrm{a}} \pm 3.127,65$ & \\
\hline \multirow[t]{2}{*}{ AST (UI/L) } & não & $1.450,30^{\mathrm{a}} \pm 349,45$ & $32-71^{* *}$ \\
\hline & $\operatorname{sim}$ & $1.557,00^{\mathrm{a}} \pm 217,17$ & \\
\hline \multirow[t]{2}{*}{ GGT (UI/L) } & não & $71,73^{\mathrm{a}} \pm 23,46$ & $3,7-31,3^{* *}$ \\
\hline & $\operatorname{sim}$ & $139,08^{b} \pm 44,11$ & \\
\hline \multirow[t]{2}{*}{ FA (UI/L) } & não & $107,10^{\mathrm{a}} \pm 36,96$ & $33-100 * *$ \\
\hline & $\operatorname{sim}$ & $207,28^{\mathrm{b}} \pm 119,00$ & \\
\hline \multirow[t]{2}{*}{$\mathrm{BT}(\mathrm{mg} / \mathrm{dL})$} & não & $0,61^{\mathrm{a}} \pm 0,50$ & $0,32-0,74^{* * *}$ \\
\hline & $\operatorname{sim}$ & $1,40^{\mathrm{b}} \pm 0,81$ & \\
\hline \multirow[t]{2}{*}{$\mathrm{BD}(\mathrm{mg} / \mathrm{dL})$} & não & $0,24^{\mathrm{a}} \pm 0,19$ & $0,01-0,34^{* * *}$ \\
\hline & $\operatorname{sim}$ & $0,83^{\mathrm{b}} \pm 0,74$ & \\
\hline \multirow[t]{2}{*}{$\mathrm{BI}(\mathrm{mg} / \mathrm{dL})$} & não & $0,36^{\mathrm{a}} \pm 0,45$ & $0,16-0,67^{* * *}$ \\
\hline & $\operatorname{sim}$ & $0,57^{\mathrm{a}} \pm 0,42$ & \\
\hline \multirow[t]{2}{*}{ Ureia $(\mathrm{mg} / \mathrm{dL})$} & não & $87,41^{\mathrm{a}} \pm 71,07$ & $5,8-35,9 * *$ \\
\hline & $\operatorname{sim}$ & $126,35^{\mathrm{a}} \pm 66,68$ & \\
\hline \multirow[t]{2}{*}{ Creatinina (mg/dL) } & não & $2,34^{\mathrm{a}} \pm 1,82$ & $1,0-2,7^{* *}$ \\
\hline & $\operatorname{sim}$ & $2,56^{\mathrm{a}} \pm 1,23$ & \\
\hline \multirow[t]{2}{*}{ PT (g/dL) } & não & $6,82^{\mathrm{a}} \pm 0,56$ & $6,5-8,6 * *$ \\
\hline & $\operatorname{sim}$ & $6,75^{\mathrm{a}} \pm 0,40$ & \\
\hline \multirow[t]{2}{*}{ Albumina (g/dL) } & não & $2,51^{\mathrm{a}} \pm 0,28$ & $2,7-4,3 * *$ \\
\hline & $\operatorname{sim}$ & $2,60^{\mathrm{a}} \pm 0,35$ & \\
\hline \multirow[t]{2}{*}{ Globulina (g/dL) } & não & $4,31^{\mathrm{a}} \pm 0,52$ & $3,0-5,0 * *$ \\
\hline & $\operatorname{sim}$ & $4,15^{\mathrm{a}} \pm 0,50$ & \\
\hline
\end{tabular}

a, b letras diferentes indicam diferença entre os grupos $(\mathrm{p}<0,05)$. * Radostits et al. (2007), ** Fagliari et al. (1998), ** Barros Filho (1995). 
vam inversão na relação linfócito:neutrófilo, duas estavam com leucocitose por neutrofilia, uma com leucocitose por linfocitose e duas não tinham alterações no leucograma. As concentrações de fibrinogênio e da proteína plasmática total estavam aumentadas em quatorze e treze dos animais examinados, respectivamente.

As vacas intoxicadas não exibiam qualquer alteração no líquor. As concentrações de proteína e de glicose, e a densidade foram, respectivamente, $32,04 \pm 9,04 \mathrm{mg} / \mathrm{dL}$, $64,66 \pm 10,61 \mathrm{mg} / \mathrm{dL}$ e 1006,18 $\pm 0,60$. A contagem de células nucleadas resultou em $2,0 \pm 1,4$ leucócitos $/ \mathrm{mm}^{3}$. Havia predomínio de células mononucleares.

Alterações extremamente significativas foram observadas para algumas variáveis bioquímicas no soro sanguíneo das vacas intoxicadas (Quadro 2). As atividades das enzimas CK e AST estavam muito acima dos intervalos de referência em todos os animais examinados. Considerando-se o limite superior fisiológico, a atividade da CK era 47 a 83 vezes maior e a atividade da AST apresentava-se 11 a 26 vezes maior. As concentrações de ureia estavam aumentadas na maioria das vacas alertas (8/10) e deprimidas $(5 / 6)$. Porém as de creatinina só estavam elevadas em cinco vacas (três alertas e duas deprimidas). Dez vacas apresentavam a concentração de albumina ligeiramente diminuída (seis alertas e quatro deprimidas). Não houve diferença para essas variáveis entre as vacas que se apresentavam alertas ou deprimidas $(\mathrm{p}>0,05)$.

A atividade da enzima GGT estava aumentada em quinze animais. Seu valor estava dentro da normalidade em apenas uma vaca, a qual permanecia alerta. A atividade da FA e as concentrações de BT e de BD estavam acima dos valores fisiológicos nos animais deprimidos e em algumas vacas que se mantiveram alertas. A concentração da BI, ao contrário, estava aumentada em apenas três das vacas examinadas. Comparadas às vacas que se apresentavam alertas, as deprimidas possuíam valores mais altos de GGT, FA, BT e BD $(\mathrm{p}<0,05)$.

Os achados de necropsia foram semelhantes nos quatro animais avaliados. As principais lesões macroscópicas foram áreas pálidas assimétricas em forma de estrias ou manchas nos músculos supraespinhoso, tríceps braquial, deltoide, infraespinhoso, longuíssimo dorsal, ileopsoas, semimembranoso, semitendinoso, glúteo, vasto lateral, vasto medial, vasto intermédio e reto femoral (Fig.2). As lesões musculares variavam de discretas a acentuadas na sua intensidade e não possuíam simetria bilateral. Havia áreas de edema entre alguns grupos musculares e no tecido subcutâneo. Em três vacas observou-se o fígado com coloração
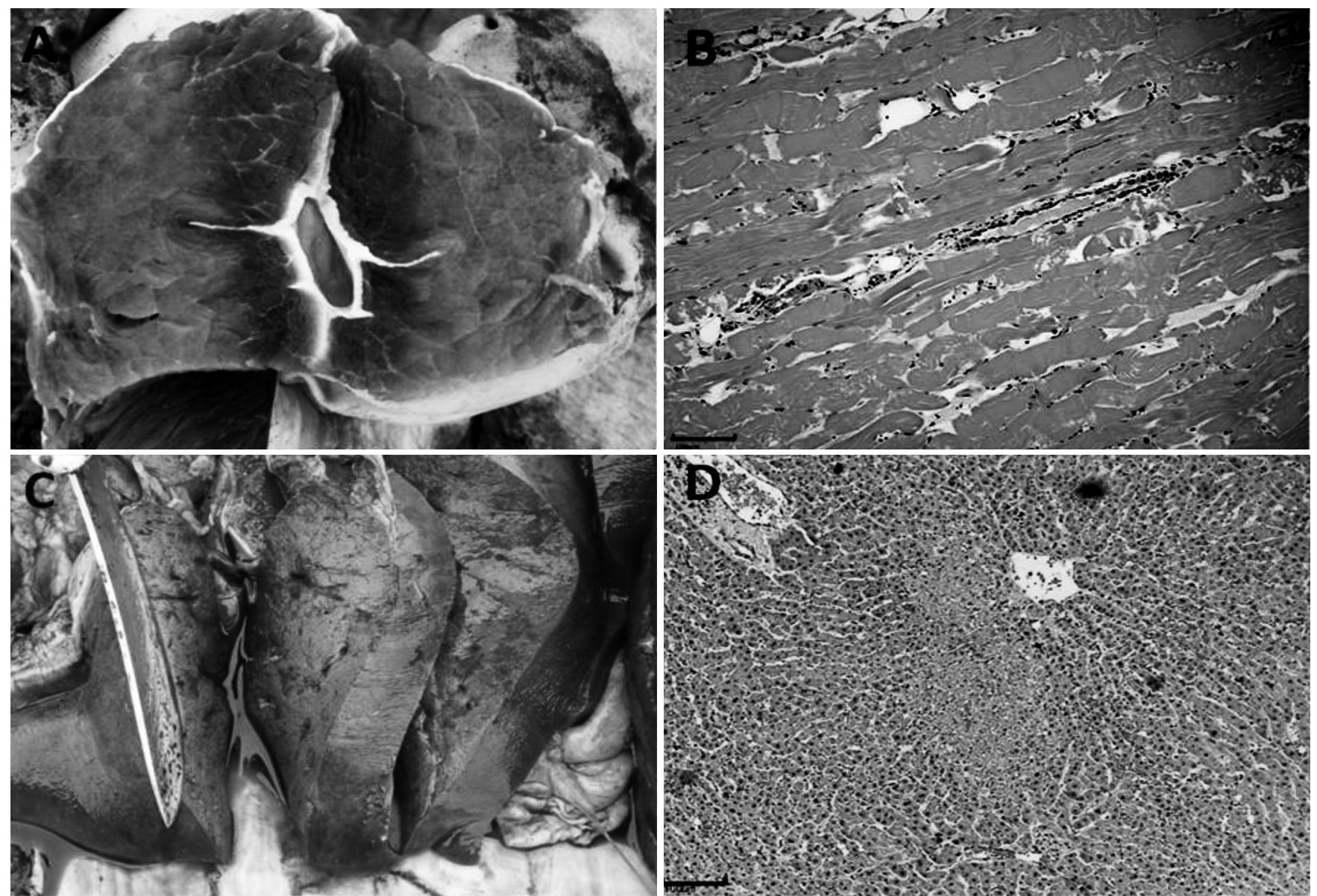

Fig.2. Quadro agudo de miopatia degenerativa causada pela ingestão de Senna obtusifolia em bovino. (A) Músculo longuíssimo dorsal mostrando área de palidez adjacente à área de coloração normal. (B) Músculo esquelético com necrose segmentar multifocal marcante com áreas de hialinização. HE, bar $100 \mu \mathrm{m}$. (C) Fígado com áreas mais pálidas entremeadas com áreas de coloração normal. (D) Corte histológico demonstrando necrose hepática multifocal paracentral. HE, bar $100 \mu \mathrm{m}$. 
não homogênea (caracterizada pela presença de manchas pálidas), com superfície irregular e com evidenciação do padrão lobular. Ao corte observaram-se áreas pálidas entremeadas com áreas de coloração normal (Fig.2). Essas três vacas tinham também icterícia. Lesão discreta no miocárdio em forma de estrias branco-amareladas foi observada em apenas um dos animais necropsiados. Não havia alterações nos demais órgãos.

Ao exame histopatológico observou-se, nos quatro animais, necrose segmentar multifocal da musculatura esquelética com infiltrado mononuclear, ruptura de fibras musculares, perda das fibras transversais, degeneração flocular, áreas de hialinização e edema intersticial, caracterizando o quadro de miopatia degenerativa (Fig.2). No fígado das três vacas que apresentavam lesão macroscópica e exibiam depressão verificou-se necrose paracentral multifocal, desorganização da estrutura trabecular, congestão sinusoidal e megalocitose de hepatócitos (Fig.2). Foram encontradas lesões renais em apenas um dos animais necropsiados consistindo em degeneração epitelial tubular, hemorragia, congestão medular, infiltrado mononuclear no córtex renal e material amorfo eosinofílico no lúmen tubular. Edema perivascular, congestão e hemorragia perivascular foram encontrados no tálamo de unicamente uma das vacas necropsiadas, a qual manifestava depressão. Na única vaca alerta que foi necropsiada observou-se, além das lesões na musculatura esquelética, necrose segmentar na musculatura cardíaca em região subendocárdica.

\section{DISCUSSÃO E CONCLUSÃO}

As evidências epidemiológicas, clínicas e patológicas indicam claramente o diagnóstico de intoxicação espontânea por Senna obtusifolia. Apesar das poucas informações disponíveis na literatura, as semelhanças com as observações anteriores de casos naturais ou experimentais dessa intoxicação em bovinos (Nicholson, Thornton \& Rimes 1977, McCormack \& Neisler 1980, Froehlich 2010) são incontestáveis. Merecem destaque também as semelhanças, em vários aspectos, entre essa intoxicação e a causada por $S$. occidentalis cujas descrições são mais numerosas (Barros et al. 1990, 1999, Raffi et al. 2003, Carmo et al. 2011, Takeuti et al. 2011).

Os índices de morbidade, mortalidade e letalidade desse surto foram respectivamente de $10 \%, 9,5 \%$ e $95 \%$, e estão de acordo com os índices encontrados por Froehlich (2010), que descreveu dois surtos de intoxicação natural por S. obtusifolia em bovinos, no estado de Santa Catarina. Nesta ocasião os índices de letalidade foram $87,5 \%$ e $100 \%$. Letalidade absoluta também foi relatada por McCormack \& Neisler (1980) nos EUA. Esses resultados confirmam que a intoxicação por essa planta é quase sempre fatal. No surto acompanhado a única vaca que se recuperou apresentou fraqueza muscular menos acentuada do que as demais, tinha dificuldade leve de locomoção e não chegou a adotar o decúbito permanente. Todas as demais vacas acabaram sendo sacrificadas posteriormente. Isso reforça a afirmação de que animais que adotam o decúbito permanente, não mais se recuperam e acabam morrendo (McCormack \& Neisler 1980).
Henson et al. (1965) observaram variações entre 1 e 6 dias para a evolução até a morte em casos de intoxicação espontânea pelas duas espécies de Senna. No surto acompanhado o período foi maior, pois alguns animais que adotaram o decúbito permaneceram alertas por um período de 5 a 9 dias, até o momento da eutanásia. Froehlich (2010) relatou que na intoxicação natural encontrou casos com evolução aguda e morte em dois dias. Outro bovino permaneceu vivo por 13 dias após adotar o decúbito permanente.

Os principais sinais clínicos observados neste surto foram fraqueza muscular, relutância ao movimento, decúbito esternal permanente, decúbito lateral permanente e mioglobinúria. Achados semelhantes foram descritos para a intoxicação espontânea (Nicholson et al. 1977, McCormack \& Neisler 1980, Froehlich 2010) e experimental (Froehlich 2010) por essa espécie de planta. Froehlich (2010) relata que a diarréia, em vários graus de intensidade, é um achado constante nos animais intoxicados natural e experimentalmente pela $S$. obtusifolia, o que só foi notado em dois animais desse surto. No restante das vacas acometidas, ao contrário, as fezes estavam ressecadas.

A mioglobinúria, caracterizada pela cor escurecida da urina, foi detectada em $37,5 \%$ das vacas no momento em que foram examinadas. Conforme relatado pelos funcionários da propriedade que acompanharam a doença em todas as suas fases, a maioria das vacas intoxicadas apresentou esse sintoma no início da evolução. Essa manifestação também foi observada por Barros et al. (1990) em bovinos intoxicados naturalmente pela semente de $S$. occidentalis. Entretanto, o mesmo não foi constatado na intoxicação experimental. Henson et al. (1965) relataram que apenas os animais com o curso clínico mais agudo da doença apresentavam mioglobinúria e, naqueles que permaneceram em decúbito por 3 a 4 dias a urina retornou à sua cor normal. Contudo, a mioglobinúria pode persistir durante os vários dias de decúbito ou pode ser intermitente, nos casos de intoxicação natural por $S$. occidentalis (Pierce \& O'Hara 1967). A vaca que permaneceu internada sob observação apresentou mioglobinúria durante os nove dias seguidos da sua evolução (Fig.1).

As alterações leucocitárias das vacas intoxicadas foram variáveis. Alterações no leucograma não foram observadas em bovinos intoxicados pelas duas espécies de Senna (Henson et al. 1965). Nas intoxicações por $S$. occidentalis já foram detectados inversão na relação linfócitos:segmentados (Schmitz \& Denton 1977) e leucocitose leve na fase final da doença, com presença de neutrófilos bastonetes que permaneceram até a morte dos animais (Rogers et al. 1979). 0 conjunto de resultados indica que o hemograma não tem utilidade diagnóstica nos casos de intoxicação por Senna em bovinos. Por outro lado, todas as vacas intoxicadas exibiam hiperfibrinogenemia, o que está de acordo com relato anterior (Schmitz \& Denton 1977), e indica a presença de processo inflamatório agudo (Jones \& Allison 2007).

As atividades aumentadas das enzimas CK e AST foram achados constantes em intoxicações naturais por S. obtusifolia (Nicholson et al. 1977, McCormack \& Neisler 1980) e naturais ou experimentais por S. occidentalis (Schmitz \& Denton 1977, Rogers et al. 1979, Barros et al. 1990, Carmo 
et al. 2011, Takeuti et al. 2011), comprovando a sua relevância para o diagnóstico. 0 aumento da atividade da enzima CK no soro é um indicativo de lesão muscular, embora não indique a origem desta lesão. As fibras da musculatura esquelética e cardíaca e o tecido nervoso são ricos nesta enzima. Assim, processos inflamatórios ou degenerativos nestes locais rompem essas células de tal maneira que as enzimas são liberadas no fluido extracelular (Cardinet 1997, Russell \& Roussel 2007). 0 aumento da atividade da enzima AST não é um achado específico para lesões musculares. Ela também está presente nos hepatócitos e em outras células, como nos eritrócitos (Russell \& Roussel 2007).

A presença de hepatopatia nas vacas intoxicadas foi indicada pelas elevações da atividade da GGT e da FA (Tennant 1997). Em comparação às vacas alertas, as deprimidas apresentavam um aumento das atividades dessas enzimas mais acentuado $(\mathrm{p}<0,05)$, assim como hiperbilirrubinemia leve devido ao aumento da concentração da bilirrubina conjugada. Esses resultados indicam que a disfunção hepática fosse mais leve nas vacas alertas e que a depressão fosse secundária à disfunção mais grave do órgão. A icterícia foi anteriormente observada por Dollahite \& Henson (1965) em bovinos intoxicados experimentalmente por $S$. occidentalis. Nessa mesma condição, Rogers, Gibson e Reichmann (1979), entretanto, não encontraram alteração nos valores da FA, da GGT e da bilirrubina.

Distintamente do relato de Nicholson et al. (1977) em bovinos intoxicados naturalmente por S. obtusifolia, 12 das 16 vacas estudadas apresentavam concentração sérica de ureia elevada. A creatinina, por outro lado, só estava aumentada em poucas vacas $(5 / 16)$ refutando a hipótese de nefropatia na maior parte desses animais. A concentração da creatinina pode se elevar quando existe lesão muscular, por ser um produto da degradação da creatina (Finco 1997). Apesar disso, na maioria das vacas intoxicadas a creatinina manteve-se próxima ao limite superior fisiológico. As cinco vacas que tinham creatinina elevada apresentavam também as concentrações mais altas de ureia. Duas delas eram deprimidas e três estavam alertas. Nessas vacas seria coerente admitir as hipóteses de azotemia renal ou pré-renal, secundária à desidratação (Russell \& Roussel 2007). De fato, uma das duas vacas azotêmicas deprimidas foi necropsiada e não exibia alterações nos rins. 0 aumento da ureia não acompanhado por elevação da creatinina foi observado em sete vacas $(85,4 \pm 44,3 \mathrm{mg} / \mathrm{dL})$. A elevação poderia ser explicada pelo catabolismo protéico aumentado consequente à baixa ingestão de alimentos (Finco 1997).

As principais lesões encontradas nos quatros bovinos necropsiados neste surto foram lesões na musculatura esquelética, dos membros posteriores e anteriores, que correspondiam a áreas pálidas assimétricas em forma de estrias ou manchas. Histologicamente estas áreas correspondiam a degeneração e necrose segmentar multifocal, com infiltrado mononuclear, necrose hialina com perda das estriações e necrose flocular. Os resultados são condizentes com os relatos anteriores de intoxicação por S. obtusifolia (Nicholson et al. 1977, McCormack \& Neisler 1980, Froehlich 2010) e são semelhantes às lesões encontradas na intoxicação natural e experimental por S. occidentalis (Dollahi- te \& Henson 1965, Henson \& Dollahite 1966, Mercer et al. 1967, Barros et al. 1990, 1999, Raffi et al. 2003, Carmo et al. 2011, Takeuti et al. 2011). Apesar de o padrão de lesão ser o mesmo nas intoxicações pelas duas espécies de Sen$n a$, algumas evidências histológicas mais específicas como a degeneração discóide (Barros et al. 1990) e a regeneração celular, caracterizada pelo aparecimento de miotubos (Barros et al. 1990, 1999. Carmo et al. 2011), não foram encontradas nas vacas intoxicadas por S. obtusifolia.

Segundo Barros (2011), a necrose da fibra dos músculos esqueléticos é, na sua maioria, de forma segmentar, ou seja, afeta apenas um segmento da fibra. A lesão inicial é a degeneração hialina, isto é, a célula muscular torna-se eosinofílica, amorfa e homogênea, sem as estrias transversais. A lesão progride para a degeneração flocular ou granular, ou seja, a fibra separa-se da membrana basal e fragmenta-se. Em 24 a 48 horas após o início da lesão, já aparece o infiltrado inflamatório, visando a fagocitar os restos necróticos das miofibras. Após os macrófagos terem fagocitado todos os debris sarcoplasmáticos, as células satélites transformadas em mioblastos arranjam-se em fileiras, unindo-se umas às outras, e produzindo os miotubos. Estes, por sua vez, enviam processos citoplasmáticos em ambos os sentidos e se fundem. A miofibra que está em regeneração é basofílica, mais estreita, não possui estrias transversais e tem seu núcleo centralizado. Nas vacas examinadas, as fases mais tardias do processo caracterizando a regeneração celular não estavam presentes, até mesmo na vaca que foi necropsiada com a evolução mais prolongada, de nove dias.

As lesões no miocárdio são pouco frequentes nas intoxicações naturais por S. obtusifolia (McCormack \& Neisler 1980) e $S$. occidentalis (Barros et al. 1999, Carmo et al. 2011). Nos casos descritos, os principais achados foram vacuolização moderada das células musculares cardíacas e fibrose focal (McCormack \& Neisler 1980, Barros et al. 1999, Carmo et al. 2011). No entanto, em intoxicações experimentais, alterações como pequenos vacúolos entre as miofibrilas com diferentes intensidades (Barros et al. 1990) e áreas de necrose do miocárdio principalmente no ventrículo esquerdo (Mercer et al. 1967), foram observadas. Além disso, uma pesquisa realizada in vitro expondo o tecido muscular cardíaco de bovino ao extrato de $S$. obtusifolia confirmou que ocorre lesão na mitocôndria (Lewis \& Shibamoto 1989). Nesse relato, apenas um animal necropsiado apresentou a lesão cardíaca. Foi necropsiado com nove dias de evolução e não apresentava alteração na frequência e no rítmo cardíacos.

As lesões renais encontradas em apenas uma das vacas estão de acordo com os achados de outros autores os quais afirmam que as lesões nesse órgão são pouco frequentes nas intoxicações por S. occidentalis (Barros et al. 1999, Raffi et al. 2003, Carmo et al. 2011, Takeuti et al. 2011).

Embora o principal problema dos animais desse surto fosse a miopatia degenerativa aguda, três dos quatro animais necropsiados tinham também hepatopatia grave. 0 efeito hepatotóxico da S. obtusifolia já foi descrito por Froehlich (2010), e um bovino que recebeu a dose de $10 \mathrm{~g} / \mathrm{kg}$ da $S$. obtusifolia por seis dias morreu devido à insuficiência hepática aguda. Lesões hepáticas degenerativas causadas 
pela intoxicação por $S$. occidentalis em bovinos foram descritas pela maioria dos autores (Dollahite \& Henson 1965, Henson \& Dollahite 1966, Mercer et al. 1967, Rogers et al. 1979, Barros et al. 1990, Takeuti et al. 2011). O'Hara, Pierce e Read (1969) atribuíram, originalmente, a lesão hepática nas intoxicações por $S$. occidentalis à insuficiência cardíaca congestiva, provocada por lesão no miocárdio. Com base em todas as evidências mais recentes, essa sugestão deve ser refutada, sendo mais coerente admitir que as duas espécies de Senna possuam princípios hepatotóxicos que produzem efeitos diretos sob o órgão. As evidências de necrose hepatocelular multifocal e das elevações mais acentuadas das atividades de GGT e FA, e das concentrações de BT e BD nas vacas que exibiam depressão, sugerem a possibilidade de que alguns animais intoxicados por S. obtusifolia possam desenvolver encefalopatia hepática. De fato, uma dessas vacas apresentava edema perivascular no tálamo.

No diagnóstico diferencial devem ser incluídas a intoxicação por S. occidentalis (Barros et al. 1990, 1999, Carmo et al. 2011), a doença do músculo branco (Barros et al. 1988), a intoxicação por antibióticos ionóforos (Barros 2007), e o botulismo (Lisbôa et al. 1996). Levando-se em conta os sinais clínicos de depressão associados à lesão hepática, deve-se estabelecer o diagnóstico diferencial com intoxicações por outras plantas hepatotóxicas e outras doenças que causem encefalopatia, como a raiva (Barros et al. 2006). Não há tratamento eficaz para animais intoxicados por $S$. obtusifolia (Nicholson et al. 1977).

Em comparação ao grande número de informações disponíveis sobre intoxicação por $S$. occidentalis, há poucos relatos de intoxicação por $S$. obtusifolia (Nicholson et al. 1977, McCormack \& Neisler 1980, Froehlich 2010), e o surto apresentado é o segundo registro científico do problema no Brasil. Os dados epidemiológicos, clínicos e patológicos são similares com os já bem conhecidos na intoxicação por $S$. occidentalis. A miopatia tóxica degenerativa é a lesão mais importante e a fraqueza muscular resultante determina o aparecimento de dificuldade de locomoção e adoção do decúbito permanente. A doença é quase sempre fatal. Apesar da S. obtusifolia ser principalmente miotóxica, os resultados desse surto demonstraram que a planta também pode causar necrose hepatocelular e determinar encefalopatia hepática em alguns bovinos intoxicados.

Agradecimentos.- À Professora Ana Odete Santos Vieira do Departamento de Biologia Animal e Vegetal da Universidade Estadual de Londrina pela classificação botânica da planta. Ao Ministério da Agricultura, Pecuária e Abastecimento/Conselho Nacional de Desenvolvimento Científico e Tecnológico pelo apoio financeiro (MAPA/CNPq processo 578645/2008-4).

\section{REFERÊNCIAS}

Ayres M.C.C., Birgel E.H., D’Angelino J.L. \& Benesi F.J. 2001. Avaliação da influência de fatores relacionados ao sexo sobre a variabilidade do eritrograma de zebuínos (Bos indicus, Linnaeus, 1758) da raça Nelore, criados no estado de São Paulo. Revta Bras. Saúde Prod. Anim. 1(2):3136.

Barros C.S.L. 2007. Intoxicação por antibióticos ionóforos, p.45-50. In: Riet-Correa F., Schild A.L., Lemos R.A.A. \& Borges J.R.J. (Eds), Doenças de Ruminantes e Equídeos. 3aㅡ ed. Pallotti, Santa Maria.

Barros C.S.L. 2011. Sistema Muscular, p.697-746. In: Santos R.L. \& Alessi A.C. (Eds), Patologia Veterinária. Roca, São Paulo.
Barros C.S.L., Barros S.S., Santos M.N. \& Metzdorf L.L. 1988. Miopatia nutricional em bovinos no Rio Grande do Sul. Pesq. Vet. Bras. 8(3/4):51-55.

Barros C.S.L., Ilha M.R.S., Bezerra Junior P.S., Langohr I.M. \& Kommers G.D. 1999. Intoxicação por Senna occidentalis (Leg. Caesalpinoideae) em bovinos em pastoreio. Pesq. Vet. Bras. 19(2):68-70.

Barros C.S.L., Pilati C., Andujar M.B., Graça D.L., Irigoyen L.F., Lopes S.T. \& Santos C.F. 1990. Intoxicação por Cassia occidentalis (Leg. Caes.) em bovinos. Pesq. Vet. Bras. 10(3/4):47-58.

Barros C.S.L., Driemeier D., Dutra I.S. \& Lemos R.A.A. 2006. Doenças do Sistema Nervoso de Bovinos no Brasil. Vallée, Minas Gerais. 207p.

Barros Filho I.R. 1995. Contribuição ao estudo da bioquímica clínica em zebuínos da raça Nelore (Bos indicus, Linnaeus 1758) criados no Estado de São Paulo. Dissertação de Mestrado em Clínica Veterinária, Faculdade de Medicina Veterinária e Zootecnia, Universidade de São Paulo, São Paulo, SP. 132p.

Cardinet G.H. III. 1997. Skeletal muscle function, p.407-440. In: Kaneko J.J., Harvey J.W. \& Bruss M.L. (Eds), Clinical Biochesmitry of Domestic Animals. $5^{\text {th }}$ ed. Academic Press, San Diego.

Carmo P.M.S., Irigoyen L.F., Lucena R.B., Fighera R.A., Komers G.D. \& Barros C.L.S. 2011. Spontaneous coffee Senna poisoning in cattle: Report on 16 outbreaks. Pesq. Vet. Bras. 31(2):139-146.

Curi P.R. 1998. Delineamentos experimentais: análise de variância, p.198211. In: Ibid. (Ed.), Metodologia e Análise da Pesquisa em Ciência Biológica. 2 a ed. Tipomic, Botucatu.

Dollahite J.W. \& Henson J.B. 1965. Toxic plants as the etiologic agent of myopathies in animals. Am. J. Vet. Res. 26(112):749-752.

Fagliari J.J., Santana A.E., Marchió W., Campos Filho E. \& Curi P.R. 1998. Constituintes sanguíneos de vacas das raças Nelore (Bos indicus) e Holandesa (Bos taurus) e de bubalinas (Bubalus bubalis) da raça Murrah durante a gestação, no dia do parto e no puerpério. Arq. Bras. Med. Vet. Zootec. 50(3):273-282.

Finco D.R. 1997. Kidney function, p.441-484. In: Kaneko J.J., Harvey J.W. \& Bruss M.L. (Eds), Clinical Biochesmitry of Domestic Animals. $5^{\text {th }}$ ed. Academic Press, San Diego.

Froehlich D.L. 2010. Intoxicação espontânea e experimental por folhas e vagens da planta Senna obtusifolia (Leguminosae) em bovinos. Dissertação de Mestrado, Universidade do estado de Santa Catarina. Centro de Ciências Agroveterinárias, Lages, SC. 38p.

Henson J.B. \& Dollahite J.W. 1966. Toxic myodegeneration in calves produced by experimental Cassia occidentalis intoxication. Am. J. Vet. Res. 27(119):947-949.

Henson J.B., Dollahite J.W., Bridges C.H. \& Rao R.R. 1965. Myodegeneration in cattle grazing cassia species. J. Am. Vet. Med. Assoc. 147(2):142-145.

Jain N.C. 1993. Shalm's Veterinary Hematology. $4^{\text {th }}$ ed. Lea and Febiger, Philadelphia. 1220p.

Jones M.L. \& Allison R.W. 2007. Evaluation of the ruminant complete blood cell count. Vet. Clin. Food Anim. 23(3):377-402.

Kaneko J.J. \& Smith R. 1967. The estimation of plasma fibrinogen and its clinical significance in the dog. California Veterinarian 21(4):21-24.

Lewis D.C. \& Shibamoto T. 1989. Effects of Cassia obtusifolia (sicklepod) extracts and anthraquinones on muscle mitochondrial function. Toxicon 27(5):519-529.

Lisbôa J.A.N., Kuchenbuck M.R.G., Dutra I.S., Gonçalves R.C., Almeida C.T. \& Barros Filho I.R. 1996. Epidemiologia e quadro clínico do botulismo epizoótico dos bovinos no estado de São Paulo. Pesq. Vet. Bras. 16(2/3):6774.

Lorenzi H. 1991. Plantas Daninhas do Brasil: terrestres, aquáticas, parasitas, tóxicas e medicinais. $2^{-}$ed. Plantarum, Nova Odessa. 268p.

Marrero E., Bulnes C. \& Perez-Ruano M. 1998. Cassia occidentalis toxicity in heifers. Toxicology Letters 95(Supl.1):153.

McCormack E. \& Neisler W.E. 1980. Cassia obtusifolia (sicklepod) toxicity in a dairy herd. Vet. Med. Small Anim. Clin. 75(12):1849-1851.

Mercer H.D., Neal F.C., Himes J.A. \& Edds G.T. 1967. Cassia occidentalis toxicosis in cattle. J. Am. Vet. Med. Assoc. 151(6):735-741.

Nicholson S.S., Thornton J.T. \& Rimes A.J. 1977. Toxic myopathy in dairy cattle caused by Cassia obtusifolia in greenchop. Bovine Pract. 12:120. 
O’Hara P.J., Pierce K.R. \& Read W.K. 1969. Degenerative myopathy associated with ingestion of Cassia occidentalis L.: Clinical and pathologic features of the experimentally induced disease. Am. J. Vet. Res. 30(12):21732180.

Pierce K.R. \& O'Hara P.J. 1967. Toxic myopathy in Texas cattle. Southwestern Vet. 20(2):179-184.

Putnam M.R., Boosinger T., Spano J., Wright J. \& Wiggins A. 1988. Evaluation of Cassia obtusifolia (sicklepod) seed consumption in Holstein calves. Vet. Hum. Toxicol. 30(4):316-318.

Radostits O.M., Gay C.C., Hinchcliff K.W. \& Constable P.E. 2007. Veterinary Medicine: A textbook of the diseases of cattle, horses, sheep, pigs and goats. $10^{\text {th }}$ ed. W.B. Saunders, London. 2065p.

Raffi M.B., Sallis E.S.V., Rech R.R., Garmatz S.L. \& Barros C.S.L. 2003. Intoxicação por Senna occidentalis em bovinos em pastoreio: relato de caso. Revta FZVA 10(1):131-136.
Rogers R.J., Gibson J. \& Reichmann K.G. 1979. The toxicity of Cassia occidentalis for cattle. Aust. Vet. J. 55(9):408-412.

Russell K.E. \& Roussel A.J. 2007. Evaluation of the ruminant serum chemistry profile. Vet. Clin. Food Anim. 23(3):403-426.

Schmitz D.G. \& Denton J.H. 1977. Senna bean toxicity in cattle. Southwestern Vet. 30(2):165-170.

Stöber M. 1993. Sistema nervoso central, p.341-362. In: Dirksen G., Gründer H.D. \& Stöber M. (Eds), Exame Clínico dos Bovinos. 3ª ed Guanabara Koogan, Rio de Janeiro.

Takeuti K.L., Raymundo D.L., Bandarra P.M., Oliveira L.G.S., Boabaid F.M., Barreto L. \& Driemeier D. 2011. Surto de intoxicação por Senna occidentalis em bovinos em pastoreio. Acta Scient. Vet. 39(1):954.

Tennant B.C. 1997. Hepatic function, p.327-440. In: Kaneko J.J., Harvey J.W. \& Bruss M.L. (Eds), Clinical Biochesmitry of Domestic Animals. $5^{\text {th }}$ ed. Academic Press, San Diego. 